\title{
Asymmetric Dimers Can Be Formed by Dewetting Half-Shells of Gold Deposited on the Surfaces of Spherical Oxide Colloids
}

\author{
Yu Lu, ${ }^{\dagger}$ Hui Xiong, ${ }^{\dagger}$ Xuchuan Jiang and Younan $\mathrm{Xia}^{*}$ \\ Department of Chemistry \\ ${ }^{\dagger}$ Department of Materials Science and Engineering \\ University of Washington, Seattle, Washington 98195 \\ *Corresponding author.E-mail: xia@chem.washington.edu
}

Mara Prentiss ${ }^{\S}$ and George M. Whitesides

${ }^{\S}$ Department of Physics

${ }^{*}$ Department of Chemistry and Chemical Biology

Harvard University, Cambridge, Massachusetts 02138 


\section{Experimental Procedure}

The monolayer of silica colloids was prepared by placing a drop of the colloidal dispersion on a flat substrate (such as silicon wafer or glass slide) and letting the solvent (in this case, water) to slowly evaporate under ambient conditions. The surface of the substrate had been treated in advance with oxygen plasma to make it hydrophilic. After water had completely evaporated, a thin film of Ti/W alloy $(\sim 5 \mathrm{~nm})$ was deposited onto the surfaces of the silica colloids as an adhesion layer using an using the MRC 822 Sputtersphere (System Control Technology, Livermore, CA). Subsequently, a thin layer of gold was sputtered on top of the Ti/W layer under a biased mode, by which gold atoms were deposited and part of them were etched away at the same time so that a dense gold film could be obtained. The substrate was then placed in a box oven and annealed in air at $700{ }^{\circ} \mathrm{C}$ for 3 hours. When gases other than air were involved, the substrate was placed in a quartz tube, and inserted into a tube furnace for thermal annealing. The resultant $\mathrm{Au} / \mathrm{SiO}_{2}$ dimers could be readily released from the supporting substrate by immersing the sample in an aqueous bath and briefly sonicated for 30 seconds. The released dimers could then be recovered from the aqueous bath by centrifugation or by filtering through a Nucleopore membrane. The use of $\mathrm{Ti} / \mathrm{W}$ alloy as the adhesion layer has already been demonstrated as a standard procedure for the preparation of thin films of noble metals on oxide surfaces. It ensures the formation of a robust film on the surface of an oxide substrate. We have also tried to directly evaporate the $\mathrm{Au}$ film without using the $\mathrm{Ti} / \mathrm{W}$ layer, and obtained $\mathrm{Au} / \mathrm{SiO}_{2}$ dimers with similar structures. In this regard, it is not really necessary to have the Ti/W alloy in order for the $\mathrm{Au}$ half-shell to dewet and become microcrystal. However, when Ti/W was used as the adhesion-promotion layer, more than $90 \%$ of the silica beads were still capped by the Au microcrystals after they had been released from the substrate. We believe that if sonication can be avoided in releasing the particles (e.g., by using substrates such as $\mathrm{NaCl}$ crystals that can be easily dissolved in water), we should be able to achieve a yield close to $100 \%$. When the gold half-shells dewetted and crystallized into the microcrystals, their final shapes might be slightly different

(probably depending on the local environment), and thus their dimensions could deviate from the expected monodispersity. Overall, the variation in size could be controlled within $5-10 \%$. 


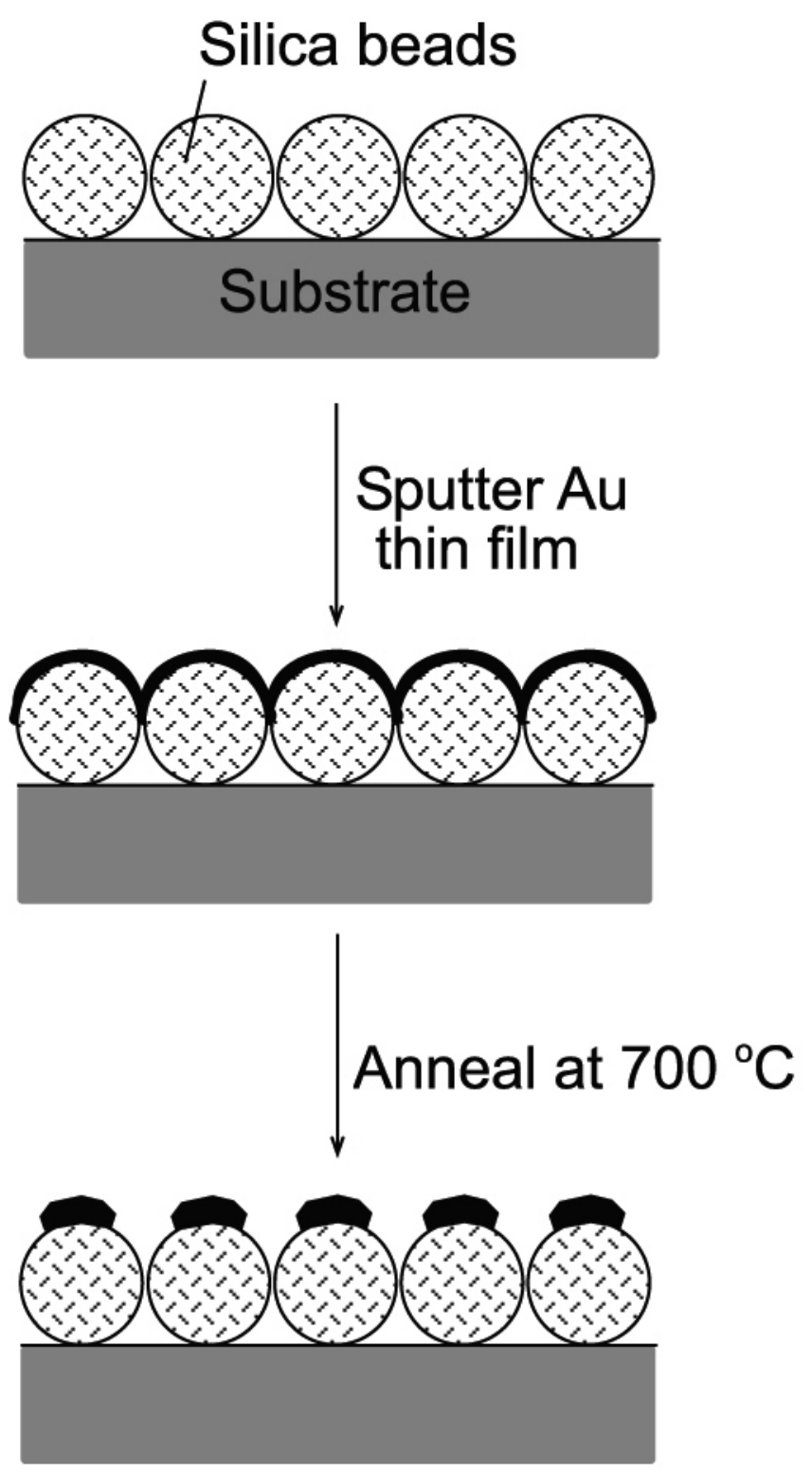

Figure S1. Schematic illustration of the experimental procedure for forming $\mathrm{Au} / \mathrm{SiO}_{2}$ dimers by dewetting Au half-shells deposited on the surfaces of spherical oxide colloids. 

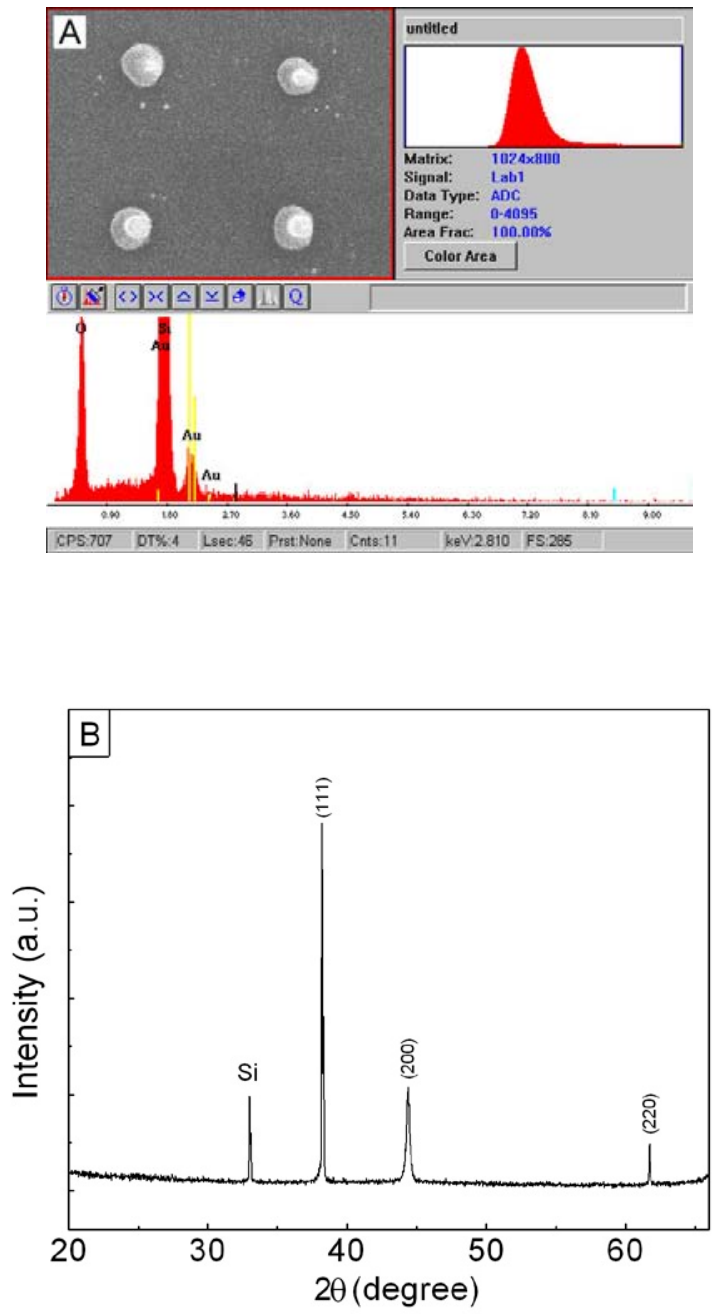

Figure S2. (A) An EDX spectrum taken from the sample shown in the inset, indicating that the composition of gold was maintained in this process. (B) A XRD pattern taken from the same sample, indicating that the gold particles were highly crystalline (the Si peak came from the underlying substrate). Since the gold microcrystals were too thick to be penetrated by electron beam, we were unable to obtain electron microdiffraction pattern from each individual crystal. As demonstrated in ref. 11 (with the use of synchrotron), gold particles prepared using a similar thermal annealing process (but on flat substrates) were, indeed, single crystals 